\title{
Using the Observer to analyze learning in virtual worlds
}

\author{
ELHANAN GAZIT and DAVID CHEN \\ Tel-Aviv University, Tel-Aviv, Israel
}

\begin{abstract}
In this study, we describe and analyze the ways in which high school students explored a virtual solar system (VSS). VSS is a nonimmersive virtual environment that affords visual manipulations of space by altering its frame of reference. The Observer software was used to code and analyze the participants' real-time free-exploration task. Two basic behaviors were identified across participants: establishing several home bases, and shifting between a still mode and a dynamic mode. Moreover, three overall exploration patterns emerged: the butterfly pattern represents a superficial mode of learning; the bee pattern represents an in-depth mode of studying the various celestialobjects; and the eagle pattern represents a global approach of studying the complex system. This virtual reality environment promises to provide a setting in which to further study consistent patterns and individual differences in exploration strategies.
\end{abstract}

\begin{abstract}
"Images are everywhere. They permeate our academic work, everyday lives, conversations and dreams" (Pink, 2001, p. 17). From birth, vision is our primary way of learning (Newcombe \& Huttenlocher, 2000; Piaget, $1929 / 1951$; Piaget \& Inhelder, 1967). It enables us to identify, recognize, and categorize objects, navigate in space, solve complex problems, and appreciate art. We use mental imagery to make plans and solve abstract problems (Arnheim, 1969; Kosslyn, 1995; Lakoff, 1987; Miller, 1987). Vision is an active cognitive process rather than a passive means of recording the environment (Palmer, 1999). By contrast, science education is based primarily on symbolic systems, which are not intuitively perceptible by most students (Chen, 1998). Hence, scientific visualizations might aid students in understanding complex scientific phenomena (Tufte, 1990). Advances in high-speed computing have facilitated the creation of 3-D simulations that represent scientific phenomena to support scientific understanding (DiSessa, 1986; Erickson, 1993). Winn (1993), Chen (1998), and Dede, Salzman, Loftin, and Ash (2000) suggested that virtual environments afford a new type of direct visual learning, which enhances knowledge building and motivation. Several researchers (Antonietti, Rasi, Imperio, \& Sacco, 2000; Youngbult, 1998) have argued that research on the potential usefulness of virtual environments in science education is in its early stages. Sánchez, Barreiro, and Maojo (2000) noted the lack of a coherent theoretical foundation in this research field. The research
\end{abstract}

This work was supported by a grant from the Sacta-Rashi Foundation for Science and Technology Education awarded to the first author. The comments of two reviewers, Vicenç Quera and Kevin G. F. Thomas, were helpful in shaping the final version of the manuscript, and we thank them for their efforts. Correspondence should be addressed to E. Gazit, School of Education, Tel-Aviv University, P. O. Box 39040, Ramat-Aviv, Tel-Aviv 69978, Israel (e-mail: gazit@ post.tau.ac.il). on virtual environments for education is focused mainly on learning outcomes, not on real-time processes. For example, Dede et al. (2000) found that subjects who observed a virtual electrical field from both a global and a local frame of reference understood the abstract concepts significantly better than did those who viewed the electrical field from a single, local frame of reference. Subjects who experienced a 3-D simulation grasped the 3-D structure of the electrical field much better than did those who studied it by using a 2-D simulation. Winn and Windschitl (2002) have identified effective and ineffective strategies used by university students to solve problems related to physical oceanography in a virtual environment.

Virtual reality environments are also used for studying human navigation and visual cognition (Kaur, Maiden, \& Sutcliffe, 1999). Darken (1996) explored navigation in four different large-scale virtual environments. The environments' attributes were found to have a significant effect on the navigation process. However, Péruch, May, and Wartenberg (1997) reported no differences in the accuracy of a navigation task in three nonimmersive virtual environments that had different computerized fields of view. It is important to note that most research on navigation in virtual environments uses environments that mimic real ones (Collinson, 1997). Lackner and DiZio (1998) argued that nonterrestrial virtual environments might cause difficulties in orientation and navigation: "Allowing the observer to view a virtual environment from perspectives not possible in a terrestrial environment such as looking straight down may similarly induce orientation illusions for some situations" (p. 115). The term cognitive map was first introduced by Tolman (1948), who found that rats hold an internal representation of the environment they explore.

In The Image of the City, Lynch (1960) concluded that humans create an internal representation of their cities 
by using five basic elements: landmarks, edges, nodes, paths, and districts. According to O' Keefe and Nadel's (1978) cognitive mapping theory, an organism successfully navigates through and within an environment using a previously formed cognitive map of that environment. When the organism observes and explores an environment, it acquires mental representations of spatial relations among stimuli in the environment, which then form a cognitive map of the environment. The cognitive map is used to generate novel search strategies within the environment. Using the cognitive mapping theory to study human spatial behavior in virtual environments, Thomas, Hsu, Laurance, Nadel, and Jacobs (2001) have shown that subjects who received training before performing a navigation task in a nonimmersive environment showed navigational advantages over subjects in the control group, who received no training. Observing the experimenter navigate in a virtual environment arena was found to be the most effective of three training procedures in teaching participants about the layout of the virtual environment and about the location of the target within that environment. Siegel (1981) suggested that spatial knowledge is acquired in three stages: (1) landmarks are coded, (2) the paths between these landmarks are learned, and (3) the paths are combined to form survey knowledge, or a cognitive map of the environment. Thrower (1999) described the Marshall Islanders' use of two types of charts for navigation in the Pacific Ocean. The rebbelib shows a broad area and uses a small scale, whereas the meddo illustrates a more restricted locality on a larger scale. Thrower claimed that rebbelib and meddo charts indicate the human need for transition between different scales of geographical knowledge. Within the context of dynamic representations, MacEachren (1995) pointed out the lack of studies in human spatial learning in dynamic geographical information systems (GISs).

Portugali (2002) introduced a different perspective on cognition in general and cognitive maps in particular. According to the synergetic inter-representation-network theory (SIRN), "Many cognitive processes, cognitive mapping included, evolve as an interaction between internal and external representations" (Portugali, 2002, p. 430). For example, a spider building its web is constructing the space along which it subsequently will be moving, in a sequential process. The structure of the web determines the routes along which the spider can move. Likewise, in several studies on rats' exploratory behavior, Golani and his coworkers (Golani, Benjamini, \& Eliam, 1993; Golani, Kafkafi, \& Drai, 1999) found that a rat under normal conditions (i.e., not induced by hunger, food, or drugs) is constructing a material external environment-a network of bases which, once constructed, determines the pattern of its subsequent exploratory behavior. Placed in a circular empty arena $6.5 \mathrm{~m}$ in diameter with a home base, the rat performs highly structured behavior consisting of two qualitatively distinct forms of movement: forward and backward. This exploratory behavior continues until the whole area is explored and full of stops, or bases, which can be differentiated according to their size, measured by the duration for which the rat stayed at them. Portugali argues that the rat constructs a cognitive map in a sequential process that evolves as a play between internal and external representations: The rat constructs a few stops and bases in a certain part of the environment; by so doing, it also memorizes them; in the next excursion it makes use of these bases, and so on. This SIRN interplay implies "a new meaning to the notion of cognitive map: The rat is actively constructing it. It not only passively perceives landmarks that exist out there in the environment, but it practically makes the landmarks - the rat is landmarking the external environment as well as its own mind/brain" (Portugali, 2002, p. 431).

Now consider the exploratory behavior of a child playing in a large-scale virtual reality environment. Much as if he were exploring a new city, the child will engage in sequential exploratory behavior by means of play between internal and external representations, but in a different experimental setup. Unlike the empty arena in Golani et al.'s (1993; Golani et al., 1999) experiments, the virtual environment is full of information. Therefore, the challenge to the child's cognitive system would be to select relevant elements in the environment (nodes, landmarks, edges, etc.) and to memorize them. Moreover, the child interacts in the virtual environment by manipulating its features. For example, while playing the computer game Simcity (Maxis), he can change the landscape by placing different objects within it. The time scale can be manipulated too, enabling the child to observe the virtual city's dynamics. Additionally, he can manipulate his point of view by zooming in or zooming out. To sum up, human-computer interaction can be seen as interplay between internal and external representation processes.

The solar system is a complex abstract concept. Nussbaum (1985) found that the cognitive difficulty of abandoning our egocentric viewpoint is a fundamental factor in misconceptions of basic astrophysical phenomena (e.g., day-night cycle, moon phases, yearly seasons). Baxter (1989), Vosniadou and Brewer (1994), Sharp (1996), and Broughton (1998) all found that many students hold intuitive mental models of these phenomena that are nonscientific.

Our main goals in the study were to identify and describe the ways in which learners explored a virtual solar system (VSS) for the first time and to study the ways in which they constructed meaning while interacting within the VSS. The importance of the current study lies in its potential contribution to the understanding of the nature of learning in dynamic virtual reality environments. First, there is an absence of coherent theory on exploration and learning within virtual worlds. Second, there is a lack of empirical studies on real-time exploration and learning within virtual environments that represent complex scientific concepts. There is a need to study the educational potential of virtual environments. Moreover, 
there is also a need to design the appropriate methodology for measuring the dynamics of learning processes within virtual worlds.

\section{METHOD}

The microdevelopment approach was selected for studying realtime processes. Microdevelopment is defined as "the process of change in abilities, knowledge, and understanding during short time spans" (Granott \& Parziale, 2002, p. 1). The defining attributes of this approach are embedded in its name: Micro pertains to short time scales - periods ranging from fractions of a second to minutes. Development indicates the evolving nature of the process, the realtime (on-line) evolution of skills and abilities of development and learning. Since the present study was an exploratory case study, no preliminary hypotheses were formulated. Case study methodology affords a systematic examination of learning processes (Stake, 1995). Young (1995), Young, Kulikowich, and Barab (1997), and Marshall (1999) suggested that qualitative and quantitative changes of the learning goals and choices made by the learner in real time could reveal the patterns of the learning processes.

\section{The VSS Environment}

The desktop VSS designed by Yair, Mintz, and Litvak (2001) is a 3-D visual simulation based on real NASA images taken from the Hubble Telescope. Using real-time rendering, the objects in VSS move in autonomous motion, on the basis of Kepler's laws of physics, regardless of the user's actions. VSS enables high levels of interactivity through the system interface by using the computer mouse. It combines symbolic information with iconic representations.

The computerized frame of reference is dynamic, affording four different observational modes: (1) The free mode enables free exploration without focusing on a preselected object. (2) With the sun-in-sight mode, the selected object becomes the virtual frame of reference. It is always shown together with reference to the sun. (3) In the stationary mode, the observer is positioned as a geocentric satellite rotating at the same rate as the object observed. The object appears to be "frozen" at the center of the computer screen while the entire "world" rotates around it. (4) In the planetary mode, the observer is locked onto the selected object as if traveling in tandem, in its orbit.

In addition, the observer can alter his or her point of view within the selected observational mode by zooming in and out, or by flying around the object in any direction (see Figure 1).

Unlike the real environment, the VSS can be visually manipulated in an infinite number of ways. This dynamic visual frame of reference enables a new learning experience. At the same time, it is also a state-of-the-art scientific visualization of astronomical knowledge.

\section{Participants and Procedure}

Ten high school students volunteered to participate in the study. Each participant watched a 4-min introductory video showing the main features of VSS. The participant was then instructed to freely explore the VSS for as long as he or she liked. The session was followed by an interview. The participant's real-time actions as they

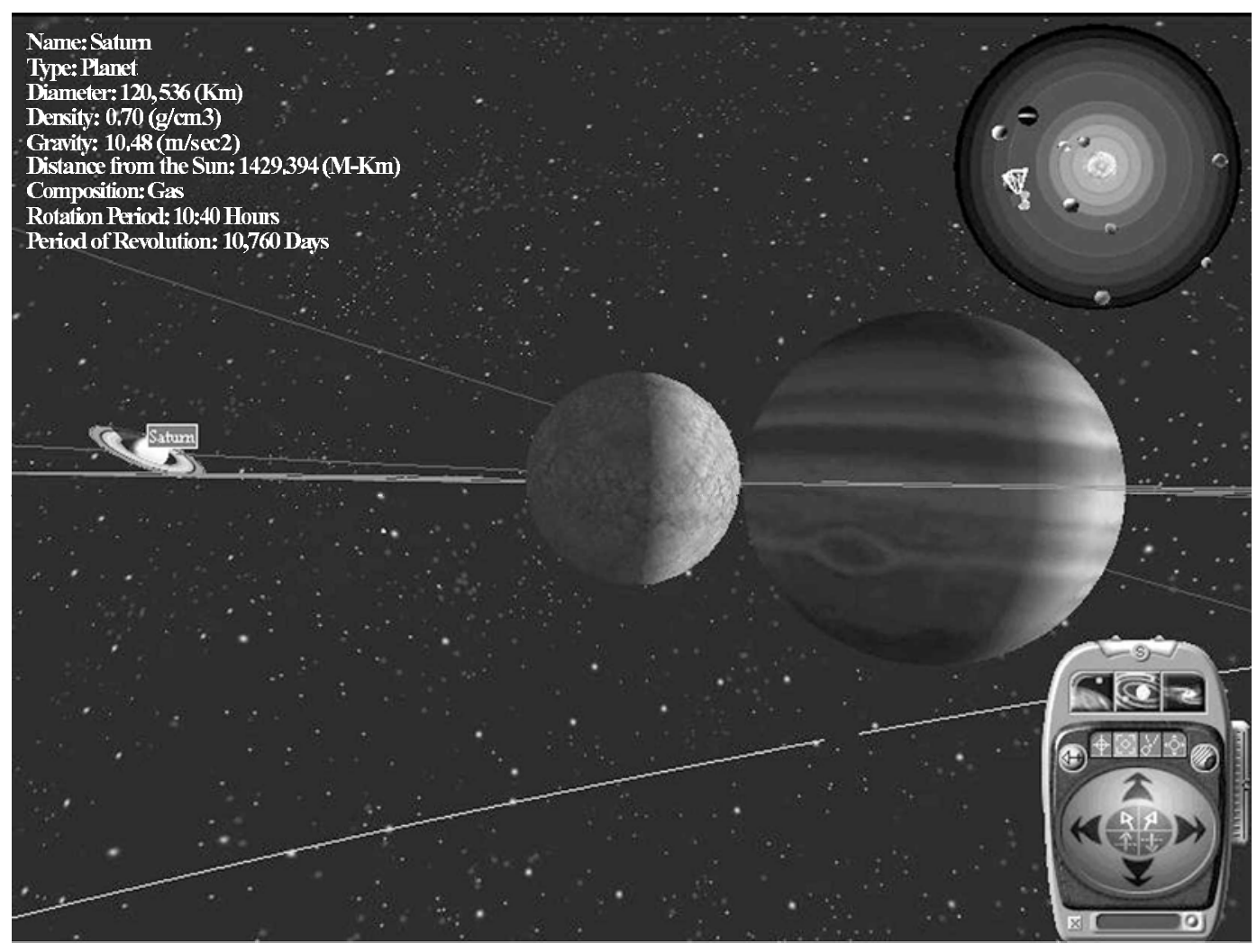

Figure 1. The virtual solar system (VSS): a screen-shot showing the moon Europa revolving around Jupiter. Saturn is located on the same plane on the left side of the screen. The planets' and moons' orbits are graphically represented, along with a 2-D map representing the nine planets in the solar system (upper right). At the lower right of the screen, the Navigator control tool appears, which affords four different observational modes. 
appeared on the computer screen, together with his or her synchronized verbal expressions, were captured.

Capturing the participant's "films" made it possible to overcome the most critical drawback of using video recordings for data collection: The fact that the recordings are usually made from the fixed point of view of the camera means, by definition, that they are not made from the participant's point of view (Granott, 1993; Harel, 1993; Henderson, 2002).

The analog videotapes were transformed to digital video format using an ATI video card. After extensive, repeated viewing of each participant's observable actions and think-aloud protocol, a coding scheme was designed. The coding scheme was used by the Observer's VideoPro software to code the raw video data. Each action of each participant was coded within $.1 \mathrm{sec}$ of resolution. During the coding process, the coding scheme was refined as new insights about the cognitive processes underlying the subjects' behaviors emerged. Finally, the coded files were analyzed by means of Observer software. This allowed the videos to be converted into meaningful data.

\section{The Coding Scheme}

The coding scheme was designed on the basis of two different sources of information: the participant's observed actions, and postsession interviews. Two levels of coding were configured: The first level was based on the participants' observed actions (Table 1), and the second level was based on their think-aloud protocol analysis.

The first coding level yielded a database of 2,575 actions. Two independent coders coded the videos, and their classif ication matched $99 \%$ of the actions.

The transcribing of the think-aloud protocol of our 10 participants yielded 1,683 sentences that formed the data bank. An extensive protocol analysis revealed five different dimensions: the interface dimension, the navigation dimension, the cognitive dimension, the affective dimension, and the scaffolding dimension. Every verbal sentence was classified in one of the five dimensions. The two independent coders coded the videos, and their classification matched $95 \%$ of the sentences. The unmatched sentences were omitted from the final analysis. For example, let us focus on the cognitive dimension. When exploring the VSS, one can observe it from different frames of reference, selecting objects that have different attributes. We found that each sentence can be classified on a five-level skill scale, which describes the development of a scientific understanding of complex systems (Table 2).

\section{RESULTS \\ Qualitative Description of Exploration Patterns}

The preliminary results are presented, with a focus on exploratory behavior within VSS. Our main goal was to describe the exploration within VSS in a free-exploration task. Two basic types of exploration behavior were identified: a dynamic mode, in which the learner interacts within a dynamic VSS, and a still mode, in which the learner interacts while VSS is kept static.

The free-exploration task usually began with the participants expressing their wish to "fly around the planets" (Eyal, age 16 years 5 months). After several minutes of dynamic-mode navigating among moving objects and changing observational modes, the still mode of behavior emerged when the participants turned the VSS into a static representation, much like a picture in a book. In this mode, the participants organized the collected symbolic and visual data and compared objects' features, making categorizations and some global generalizations (e.g., "Mars rotates around its axis much like Earth. Its day-night cycle is almost like ours on Earth"; Ori, age 15 years 7 months). Five participants reported experiencing cognitive conflict regarding their own mental model of the solar system: "What? I thought that the more distant the planet is from the sun, the faster it spins on its axis" (Ori). "Why are the distant planets [from the sun] much bigger? And why are the close planets like Venus and Mercury made of rocks? I thought it had to be the other way around" (Sivan, age 15 years 9 months). Following this, the dynamic mode pattern reappeared several times during the spontaneous exploration task. Seven of the participants made generalizations, seemingly changing their mental models of the solar system. During the exploration, they expressed their emotions: "The more we explore space, we see how small we are, that we are not so important in this vast universe. Suddenly our problems seem less important, in proportion to the other very important things that occur in the universe" (Ann, age 15 years 7 months).

In the dynamic mode, three exploration patterns were found: The butterfly pattern, the bee pattern, and the eagle pattern. The main differences between these dynamic patterns of exploration were in terms of the preferred observational mode, the pace and duration of object exploration, the "scale level" of exploration (local vs. global), and the frequency of attending symbolic data. In the butterfly pattern, the participant tends to observe in a free observational mode, jumping from object to object and paying little attention to their details. In the

Table 1

Observed VSS Real-Time Actions

\begin{tabular}{|c|c|}
\hline Observed Action & Definition \\
\hline Selecting an object & The participant selects an object by clicking the mouse or pointing the mouse's cursor on it. \\
\hline $\begin{array}{l}\text { Selecting a computerized } \\
\text { observational mode }\end{array}$ & The participant selects an observational mode by clicking on the Navigator. \\
\hline $\begin{array}{l}\text { Altering point of view within a } \\
\text { computerized observational mode }\end{array}$ & $\begin{array}{l}\text { The participant alters his or her computerized point of view by pointing the mouse's cursor in different } \\
\text { directions and clicking. }\end{array}$ \\
\hline Passing between objects & $\begin{array}{l}\text { The participant passes between objects by clicking on a 3-D object, by clicking on a 2-D object, or by } \\
\text { selecting it from the toolbar list. }\end{array}$ \\
\hline Altering the system's pace & The participant stops or enhances the object's motion by changing the pace arrow in the Navigator. \\
\hline
\end{tabular}


Table 2

Skill Levels of Scientific Understanding of Complex Systems: The Case of the Solar System

\begin{tabular}{cll}
\hline Level & \multicolumn{1}{c}{ Definition } & \multicolumn{1}{c}{ Example of Student Verbal Expression } \\
\hline 1 & $\begin{array}{l}\text { Declarative local level: Describing the attributes of a single } \\
\text { object, focusing on the system's parts one at a time }\end{array}$ & "We can see that there are many holes on the moon's surface." \\
2 & $\begin{array}{l}\text { Procedural local level: Comparing object attributes, } \\
\text { generalizing from the local perspective }\end{array}$ & $\begin{array}{l}\text { "I see that the surface of Mars is red, so I hypothesize that it's hot } \\
\text { on its surface." } \\
\text { "Nine planets revolving around the sun." "Planet Jupiter has four } \\
\text { moons." } \\
\text { "I hypothesize that there is a connection between the position of the } \\
\text { object in space and its composition." } \\
\text { (e.g., symbolic, visual) }\end{array}$ \\
& $\begin{array}{l}\text { Procedural global level: Comparing objects' features from a } \\
\text { global perspective, making hypotheses }\end{array}$ & $\begin{array}{l}\text { Higher procedural global level: Understanding the system's } \\
\text { dynamics and principles, making generalizations }\end{array}$ \\
\hline
\end{tabular}

bee pattern, the participant tends to observe in a planetary observational mode, zooming in to examine objects and establishing a home base (i.e., returning to the same object in the course of the task). The participant focuses on the system's components. The eagle pattern involves a tendency to observe in a free observational mode and in a sun-in-sight observational mode, since the participant observes the solar system globally. No clear correlation was found between the students' exploration patterns and their prior mental model of the solar system. Six participants shifted among the three dynamic patterns throughout the session, whereas 4 others maintained a single pattern.

A time-event plot generated by Observer provided insight into the interaction complexity within the VSS. Over 200 behaviors were coded for each participant, regardless of the time spent on the task. Coding in consequential states enabled capture of the multidimensional na- ture of the learning process during the free-exploration task (see Figure 2).

Figure 2 shows a 2-D time-event plot of Ori's (age 15 years 7 months) exploration behavior, as generated by Observer. The sequence and duration of the coded behaviors can be seen on the time scale. Line 1 shows the selected system pace. Light gray represents a dynamic mode of VSS. Black represents a still mode. The other lines represent the objects selected during the exploration pattern. The objects are arranged according to their distance from the sun. As can be seen, Ori entered the VSS by selecting to view an asteroid. Then, she moved on to follow the comet orbit (Line 16) in a free observational mode (not shown in the figure). She established two home bases: the comet orbit (Line 16) as seen from the subsystem perspective (Line 17) and the 2-D map (Line 22) from which she collected additional global visual information on the spatial configuration of the plan-

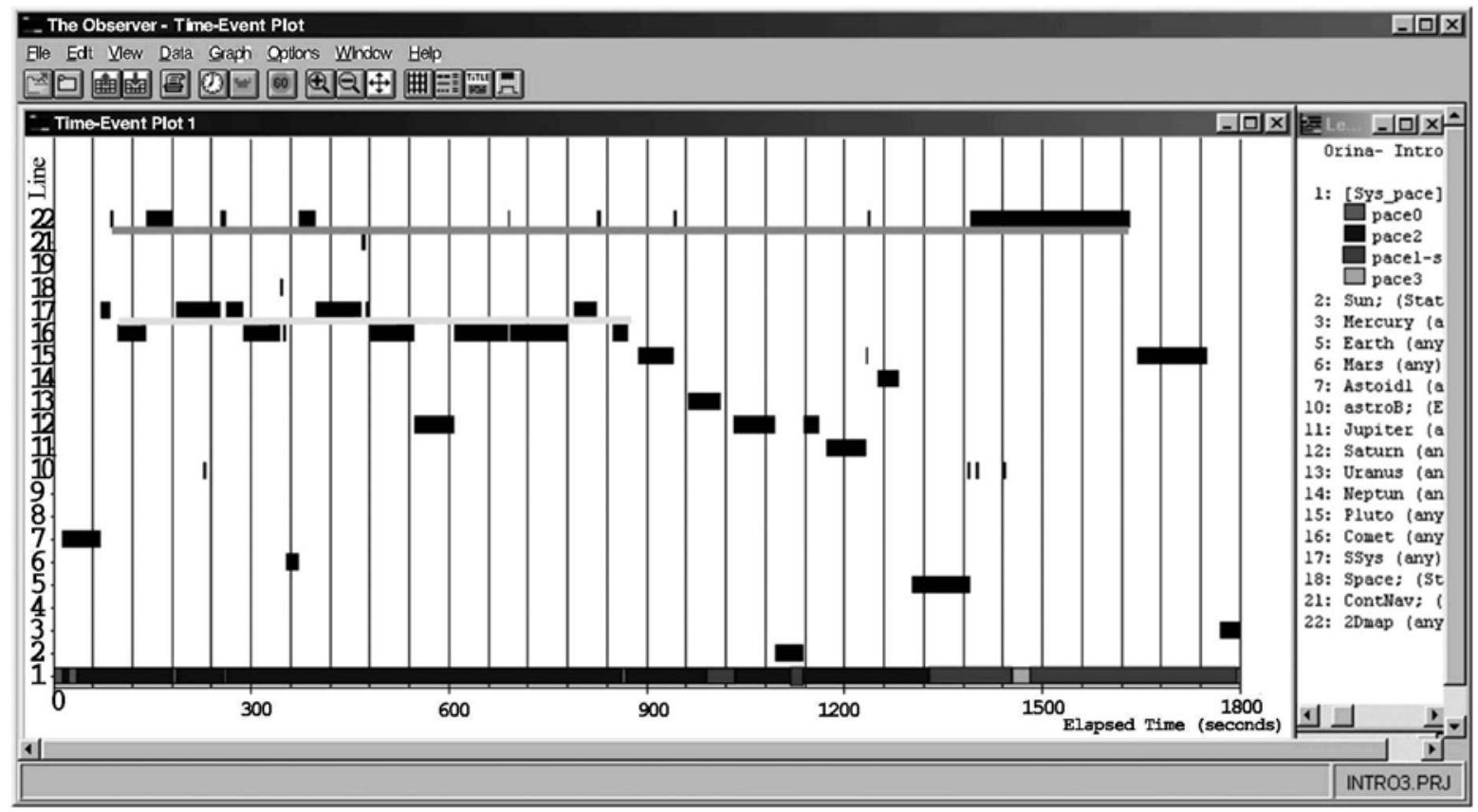

Figure 2. Time-event plot of Ori's (age 15 years 7 months) free-exploration task. 


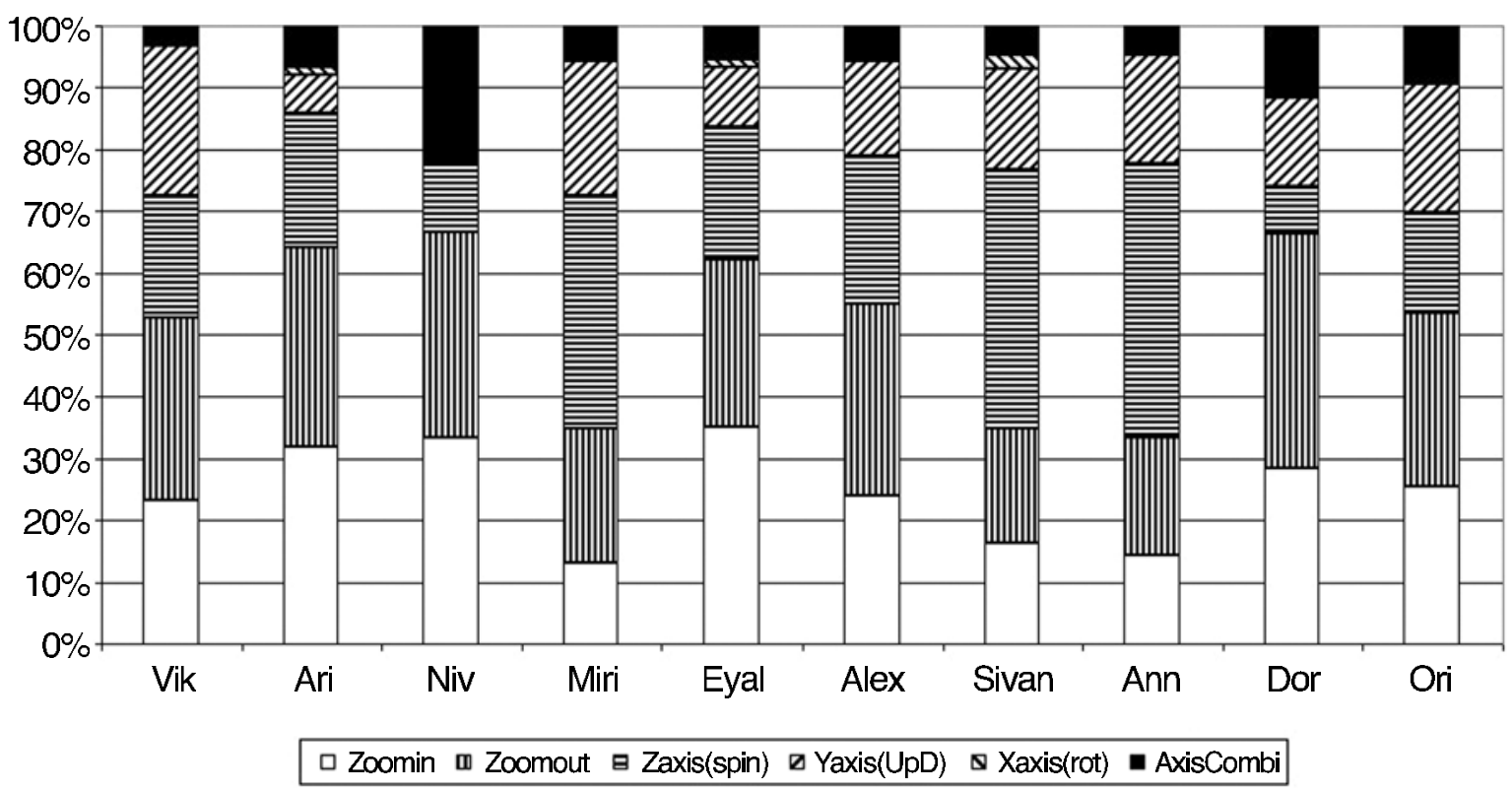

Figure 3. Relative proportions of the altering-point-of-view frequencies within an individual framework $(N=700)$.

ets. By observing the system from a global frame of reference in a free observational mode, Ori demonstrated the eagle exploration pattern. Following that, Ori demonstrated yet a different pattern, moving between the distant planets such as Pluto (Line 15), Saturn (Line 12), Uranus (Line 13), and Jupiter (Line 11). She observed their moons from planetary and stationary observational modes. Additionally, she altered her point of view by zooming in and flying around the moons to examine their surfaces and colors. In so doing, Ori demonstrated the bee exploration pattern.

All 10 participants established several home bases during the exploration task. Two to three objects selected at the beginning of the session were reselected several

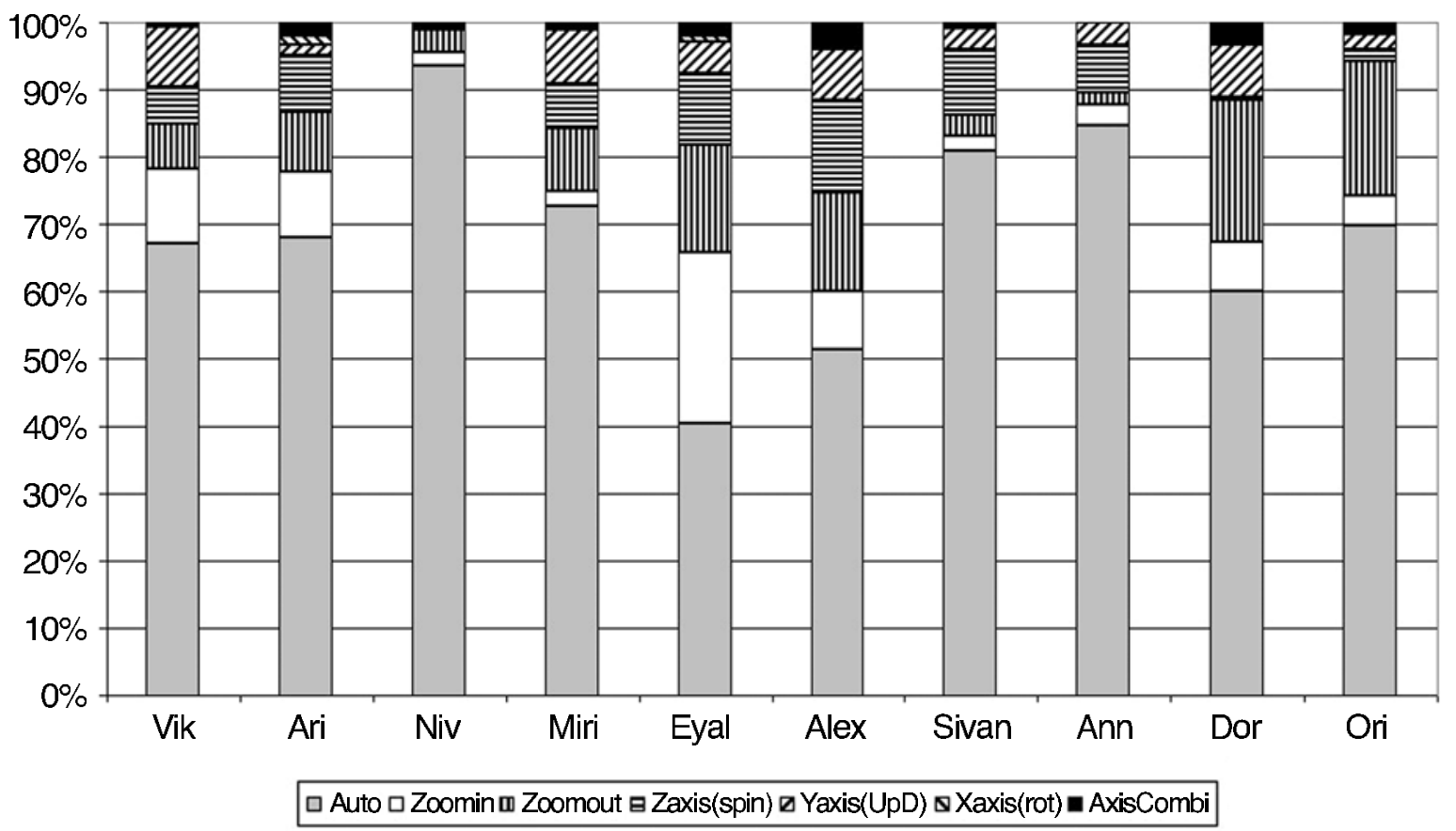

Figure 4. Relative proportions of altering-point-of-view duration within an individual framework. 
times during the free-exploration task. Another commonality was found regarding the relative proportions of the altering-point-of-view patterns within individual framework modes (see Figure 3).

Figure 3 shows that all 10 participants preferred either to zoom in on an object or to zoom out away from it. Altering the point of view in a combination of directions was found to be less frequent than altering the point of view in a single direction, such as spinning around the object's $z$-axis.

The histograms presented in Figure 4 show that the relative proportions of the durations of 2 participants' active control over the system's point of view ranged from less than $10 \%$ (Niv, age 16 years 9 months) to $60 \%$ (Eyal, age 16 years 5 months). The active altering-pointof-view duration of the other participants varied within that range. For example, Eyal performed a butterfly pattern throughout the exploration task, spending most of his time in free observational mode, altering his point of view constantly and passing between the objects. By contrast, Niv moved between a bee pattern and an eagle pattern, spending less time altering his point of view within his preferred planetary observational mode. Likewise, individual differences were found regarding other aspects of the interaction. For example, the interaction pace ranged from a high of 11.11 actions per minute (Eyal) to a low of 5.19 actions per minute (Niv).

\section{DISCUSSION}

In this study, the ways that 10 high school students interacted with a new VSS for the first time was described. By using Observer software, a rich fabric and dynamics of learning could be identified. A systematic analysis of the learning process has shown the categorization of this complex behavior into well-defined patterns or schemas. The most evident pattern involves the emergence of several home bases. This possibly serves as a stable anchor for spatial organization in a dynamic visual representation. The second pattern identified was the move between a dynamic and a static mode. The learner might use this to take control of the flow of information. This human-computer interaction seems to support the SIRN by the actual demonstration of interplay between internal and external representations. By altering his or her point of view, the learner could gain additional visual information about a given object. Shifting between local and global frames of reference might contribute to the creation of multidimensional perspectives on the phenomena under study. Moreover, within the shared patterns, three different learning styles could be identified. In the bee pattern, the learner tends to focus on the system's parts; in the eagle pattern, the learner examines the system's global attributes; in the butterfly pattern, the learner focuses on the act of moving and navigating in the 3-D environment. These metaphors capture a consistent approach to deciphering the complex human-computer interaction phenomena under study.
In conclusion, studying the dynamics of real-time virtual environment interactions was possible through the use of Observer. By incorporating new digital technologies into known methodologies, we can gain new insights into the complex nature of learning within dynamic virtual environments. Additional research will enhance our understanding of the dynamics of learning within virtual environments.

\section{REFERENCES}

Antonietti, A., Rasi, C., Imperio, E., \& SAcco, M. (2000). The representation of virtual reality in education. Education \& Information Technologies, 5, 317-327.

ArnheIm, R. (1969). Visual thinking. Berkeley: University of California Press.

BAXTER, J. H. (1989). Children's understanding of familiar astronomical events. International Journal of Science Education, 11, 502-513.

Broughton, M. (1998). Alternative frameworks amongst University of Plymouth astronomy students. In E. Gougenheim, D. A. Pierce, \& N. McNally (Eds.), Recent advances in astronomyteaching (pp. 111117). Cambridge: Cambridge University Press.

Chen, D. (1998). The impact of technology on the relations between children and knowledge. Eureka Journal of Science \& Technology, 6, 8-15 (in Hebrew).

Collinson, A. (1997). Virtual worlds. Cartographic Journal, 34, 117 124.

DARKEN, R. P. (1996). Wayfinding in large-scale virtual worlds. Unpublished doctoral dissertation, George Washington University, Washington, DC

Dede, C., Salzman, M., Loftin, B., \& Ash, K. (2000). The design of immersive virtual learning environments: Fostering deep understandings of complex scientific knowledge. In M. J. Jacobson \& R. B. Kozma (Eds.), Innovations in science and mathematics education (pp. 361-413). Mahwah, NJ: Erlbaum.

DiSessa, A. (1986). Artificial worlds and real experience. Instructional Science, 14, 207-227.

ERICKSON, T. (1993). Artificial realities as data visualization environments. In A. Wexelblat (Ed.), Virtual reality: Applications and explorations (pp. 1-22). Boston: Academic Press.

Golani, I., Benjamini, Y., \& Eliam, D. (1993). Stopping behavior: Constraints on exploration in rats. Behavior \& Brain Research, 53, 21-33.

Golani, I., KafKaFI, N., \& Drai, D. (1999). Phenotyping stereotypic behavior: Collective variables, range of variation and predictability. Applied Animal Behavior Science, 65, 191-220.

GranotT, N. (1993). Through the camera lens: Video as a research tool. Spontaneous inquiry and phases in knowledge construction. In I. Harel \& S. Papert (Eds.), Constructionism (pp. 319-326). Norwood, NJ: Ablex.

Granott, N., \& Parziale, J. (Eds.) (2002). Microdevelopment: Transition processes in development and learning. Cambridge: Cambridge University Press.

HAREL, I. (1993). The silent observer and holistic note-taker: Using video for documenting a research project. In I. Harel \& S. Papert (Eds.), Constructionism (pp. 327-343). Norwood, NJ: Ablex.

Henderson, A. (2002, August). Evolving understanding: Using video records in interaction analysis. Paper presented at Measuring Behavior 2002, 4th International Conference on Methods and Techniques in Behavioral Research, Amsterdam.

Kaur, K., Maiden, N., \& Sutcliffe, A. (1999). Interacting with virtual environments: An evaluation of a model of interaction. Interacting with Computers, 11, 403-425.

KoSSLYN, M. S. (1995). Image and brain: The resolution of the imagery debate. Cambridge, MA: MIT Press.

LACKNER, J. R. \& DiZio, P. (1998). Spatial orientation as a component of presence: Insights gained from nonterrestrial environments. Presence, 7, 108-115.

LAKoff, G. (1987). Women, fire, and dangerous things. Chicago: University of Chicago Press. 
LYNCH, K. (1960). The image of the city. Cambridge, MA: MIT Press. MACEACHREN, A. M. (1995). How maps work: Representation, visualization, and design. New York: Guilford.

MARSHALl, G. (1999). Exploring assessment. Education \& Information Technologies, 4, 311-327.

MiLleR, A. I. (1987). Imagery in scientific thought. Cambridge, MA: MIT Press.

Newcombe, N. S., \& Huttenlocher, J. (2000). Making space. Cambridge, MA: MIT Press.

Nussbaum, J. (1985). The earth as a cosmic body. In R. Driver (Ed.), Children's ideas in science. Milton Keynes, U.K.: Open University Press.

O'KeEFe, J., \& NADEL, L. (1978). The hippocampus as a cognitive map. Oxford: Oxford University Press.

PALMER, S. E. (1999). Vision science from photons to phenomenology. Cambridge, MA: MIT Press.

Péruch, P., May, M., \& Wartenberg, F. (1997). Homing in virtual environment: Effects of field of view and path layout. Perception, 26 301-311.

Piaget, J. (1951). The child's conception of the world (J. Tomlinson \& A. Tomlinson, Trans.). New York: Humanities Press. (Original work published 1929)

Piaget, J., \& InHELder, B. (1967). The child's conception of space (F. J. Langdon \& J. L. Lunzer, Trans.). New York: Norton.

PINK, S. (2001). Doing visual ethnography. London: Sage.

Portugali, J. (2002). The seven basic propositions of SIRN (Synergetic Inter-Representation Networks). Nonlinear Phenomena in Complex Systems, 5, 428-444.

Sánchez, A., Barreiro, J. M., \& Maojo, V. (2000). Design of virtual reality systems for education: A cognitive approach. Education \& Information Technologies, 5, 345-362.

SHARP, J. G. (1996). Children's astronomical beliefs: A preliminary study of year 6 children in southwest England. International Journal of Science Education, 18, 685-712.

SIEGEL, A. W. (1981). The externalization of cognitive maps by children and adults: In search of ways to ask better questions. In L. S. Liben, A. Patterson, \& N. Newcombe (Eds.), Spatial representation and be- havior across the lifespan: Theory and application (pp. 167-194). New York: Academic Press.

StAKe, R. E. (1995). The art of case study research. New York: Sage.

Thomas, K. G. F., Hsu, M., Laurance, H. E., Nadel, L., \& Jacobs, W. J. (2001). Place learning in virtual space III: Investigation of spatial navigation training procedures and their application to $\mathrm{MRI}$ and clinical neuropsychology. Behavior Research Methods, Instruments, \& Computers, 33, 21-37.

Thrower, N. J. W. (1999). Maps and civilization. Chicago: University of Chicago Press.

Tolman, E. C. (1948). Cognitive maps in rats and men. Psychological Review, 55, 189-208.

Tufte, E. R. (1990). Envisioning information. Cheshire, CT: Graphics Press.

VosNiAdOU, S., \& BREWER, W. F. (1994). Mental models of the day/night cycle. Cognitive Science, 18, 123-183.

WINN, W. [D.] (1993). A conceptual basis for educational applications of virtual reality (HITL Tech. Rep. No. R-93-9). Seattle: University of Washington, Human Interface Technology Laboratory.

Winn, W. D., \& Windschitl, M. (2002, April). Strategies used by university students to learn aspects of physical oceanography in a virtual environment. Paper presented at the 83rd Annual Meeting of the American Educational Research Association, New Orleans.

YAIR, Y., MinTz, R. \& LiTVAK, S. (2001). 3D-virtual reality in science education: An implication for astronomy teaching. Journal of Computers in Mathematics \& Science Teaching, 20, 293-305.

YounG, M. [F.] (1995). Assessment of situated learning using computer environments. Journal of Science Education \& Technology, 4, 89-96.

Young, M. F., Kulikowich, J. M., \& Barab, S. A. (1997). The unit of analysis for situated assessment. Instructional Science, 25, 133-150.

YoungBult, C. (1998). Educational uses of virtual reality technology. IDA Document D-2128. Alexandria, VA: Institute for Defense Analyses.

(Manuscript received October 1, 2002; revision accepted for publication June 2, 2003.) 